\title{
Chromospheric evaporation in sympathetic coronal bright points
}

\author{
Q. M. Zhang ${ }^{1,2}$ and H. S. Ji ${ }^{1}$ \\ ${ }^{1}$ Key Laboratory for Dark Matter and Space Science, Purple Mountain Observatory, CAS, 210008 Nanjing, PR China \\ e-mail: zhangqm@pmo.ac.cn \\ 2 Key Lab of Modern Astronomy and Astrophysics, Ministry of Education, PR China
}

Received 17 May 2013 / Accepted 26 July 2013

\begin{abstract}
Context. Chromospheric evaporation is a key process in solar flares that has been extensively investigated using spectroscopic observations. However, direct soft X-ray (SXR) imaging of the process is rare, especially in remote brightenings associated with the primary flares that have recently attracted a great deal of attention.

Aims. We intend to find the evidence for chromospheric evaporation and figure out the cause of the process in sympathetic coronal bright points (CBPs), i.e., remote brightenings induced by the primary CBP.

Methods. We utilised the high-cadence and high-resolution SXR observations of CBPs from the X-ray Telescope (XRT) aboard the Hinode spacecraft on 2009 August 23.

Results. We discovered a thermal conduction front propagating from the primary CBP (hereafter BP1) to the first of the sympathetic CBPs (hereafter BP2) that is $60^{\prime \prime}$ away from BP1. The apparent velocity of the thermal conduction is $\sim 138 \mathrm{~km} \mathrm{~s}^{-1}$. Afterwards, hot plasma flowed upwards into the loop connecting BP1 and BP2 at a speed of $\sim 76 \mathrm{~km} \mathrm{~s}^{-1}$, a clear signature of chromospheric evaporation. Similar upflow was also observed in the loop connecting BP1 and the other sympathetic CBP (hereafter BP3) that is 80" away from BP1, though less significant than BP2. The apparent velocity of the upflow is $\sim 47 \mathrm{~km} \mathrm{~s}^{-1}$. The thermal conduction front propagating from BP1 to BP3 was not well identified except for the jet-like motion also originating from BP1.

Conclusions. We propose that the gentle chromospheric evaporation in the sympathetic CBPs were caused by thermal conduction originating from the primary CBP.
\end{abstract}

Key words. Sun: corona - Sun: activity - Sun: X-rays, gamma rays

\section{Introduction}

Solar flares are transient energy releases via magnetic reconnection accompanied by localized plasma heating and particle accelerations (Svestka 1976; Gan et al. 1991; Ding \& Fang 2001; Ji et al. 2006, 2007, 2008; Guo et al. 2008; Fletcher et al. 2011; Ning \& Cao 2011; Shibata \& Magara 2011; Chen 2012; Hao et al. 2012; Su et al. 2013). According to the standard flare model, known as the CSHKP model (Carmichael 1964; Sturrock 1966; Hirayama 1974; Kopp \& Pneuman 1976), substantial nonthermal electrons (10-100 keV) propagate downwards along the reconnected magnetic field lines and collide with the dense chromosphere, creating impulsive hard X-ray (HXR) emissions and thermalizing the local plasma to 1-10 MK. Meanwhile, thermal conduction propagates downwards from the super-hot reconnection region. The overpressure of the chromosphere propels hot plasma into the tenuous coronal loops that emit strong emissions in soft X-ray (SXR), a process called chromospheric evaporation (Neupert 1968; Fisher et al. 1985a, 1985b, 1985c; Emslie et al. 1992; Allred et al. 2005). Fisher et al. (1985b) divided chromospheric evaporation into two types according to the level of the energy flux. If the energy flux exceeds a critical value of $\sim 10^{10} \mathrm{erg} \mathrm{cm}^{-2} \mathrm{~s}^{-1}$, then explosive evaporation takes place accompanied by blueshifts at speed of hundreds of $\mathrm{km} \mathrm{s}^{-1}$ in the emission lines formed in the coronal temperature and redshifts at speed of tens of $\mathrm{km} \mathrm{s}^{-1}$ in the emission lines formed in the transition region and upper chromosphere (Brosius \& Holman 2007). Otherwise, gentle evaporation takes place accompanied by blueshifts at speed of tens of $\mathrm{km} \mathrm{s}^{-1}$ in all emission lines (Brosius \& Phillips 2004; Brosius \& Holman 2009; Berkebile-Stoiser et al. 2009). Both nonthermal electrons and thermal conduction have been reported to play an important role in the gentle evaporation (Milligan et al. 2006; Milligan 2008). Up to now, chromospheric evaporation has been observed by the space-borne spectroscopic imagers in extreme ultraviolet wavebands (EUV; Chen \& Ding 2010; Li \& Ding 2011) and HXR (Liu et al. 2006; Ning et al. 2009). In SXR, however, the process has seldom been reported owing to the low spatial resolution, time cadence, and temperature sensitivity of the previous telescopes (Silva et al. 1997). Thanks to the state-of-theart X-ray Telescope (XRT; Golub et al. 2007) aboard Hinode (Kosugi et al. 2007), it has become possible to seek for direct evidence of chromospheric evaporation. Nitta et al. (2012) for the first time observed clear chromospheric evaporation upflows arising almost symmetrically from the footpoints of magnetic loops. Among the 13 small transient brightenings observed by Hinode/XRT, nearly $46 \%$ had an average apparent velocity of $100 \mathrm{~km} \mathrm{~s}^{-1}$, and the other $23 \%$ were much faster. Nevertheless, magnetic reconnections that lead to the evaporation were not recognized in their limited sample, and the cause of evaporation (thermal conduction or nonthermal electrons) was not clarified. Milligan (2008) showed apparent flow of hot material 
along the GOES B-class flare loop. The absence of detectable HXR emission coupled with lower upflow velocities suggests gentle chromospheric evaporation driven by thermal conduction.

Because of the complexities and interconnections of magnetic fields in the solar atmosphere, flares may not occur independently. Occasionally, a flare in the primary region may induce another one in a remote region, which is called a sympathetic flare (Richardson 1951). The mechanism of energy transport between the two regions has been extensively investigated. It was proposed that the possible driving agents could be energetic particles, shock waves, and thermal conduction (Machado et al. 1988). For example, Zhang et al. (2000) studied both the magnetic topology and the time delay between the initial and sympathetic flares, finding that heat conduction is responsible for the sympathetic flare. For flares associated with coronal mass ejections (CMEs), the erupting flux rope and its envelope magnetic field may reconnect with the overlying field lines, producing a bright ribbon (or sympathetic flare) in a remote site (Wang 2005). Interestingly, Brosius \& Holman (2007) discovered explosive chromospheric evaporation in a remote solar flare-like transient. Wang \& Liu (2012) found remote brightenings at a region far from the circular-ribbon flares. Deng et al. (2013) also discovered a circular-ribbon flare with a remote brightening that is predicted in three-dimensional fan-spine reconnection.

Coronal bright points (CBPs) are long-lived $(8 \mathrm{~h})$ small-scale $\left(10^{\prime \prime}-40^{\prime \prime}\right)$ brightenings in the lower corona (Golub et al. 1977; Priest et al. 1994; Tian et al. 2008). Like big flares, they are also believed to be heated by magnetic reconnection. Sometimes, recurrent strong flashes appear in CBPs with significant brightness enhancement (Zhang et al. 2012). If a CBP flash occurs at the footpoint of a large-scale magnetic loop, chances are that another transient brightening, i.e., CBP, will be induced at the remote site of the large-scale loop because of the energy flux transported by nonthermal electrons or thermal conduction along the loop, which is termed sympathetic CBP. These events and the associated chromospheric evaporation, to our knowledge, have not been investigated yet. In this letter, we report our detection of chromospheric evaporation in sympathetic CBPs observed by Hinode/XRT. In Sect. 2 we describe the data analysis and show the results. Discussion and conclusion are presented in Sect. 3.

\section{Data analysis and results}

The XRT instrument has nine filters that achieve a wide temperature coverage from 0.3 MK to 30 MK. During 2009 August 22, 23, it pointed to a high-latitude region close to the north polar coronal hole using the C_poly filter that has maximum temperature response at $\log T \approx 6.95$. The partial-frame $\left(384^{\prime \prime} \times 384^{\prime \prime}\right)$ observations lasted for more than $10 \mathrm{~h}$ from 15:00 UT on August 22 to 01:30 UT on August 23 with time cadence of $32 \mathrm{~s}$ and spatial resolution of 2'.06. During the observations, a bright point in the field of view experienced several strong flashes, the last of which occurred around 01:00 UT on August 23 and triggered another two bright points.

Figure 1 shows four snapshots of the SXR images that represent the four stages of the evolution of the CBPs. We note that the color is reversed so that the dark regions in the panels stand for bright features in the corona. In Fig. 1a, the main CBP (hereafter BP1) exists as a tiny bright loop. The distance of the footpoints of the loop is about $15^{\prime \prime}$. It started to increase slowly in size and brightness from 00:56 UT on August 23. The quiescent square region, i.e., BG, within the box is the background with slight intensity fluctuation. The size and intensity of BP1 increased rapidly from 01:00 UT and reached maximum at

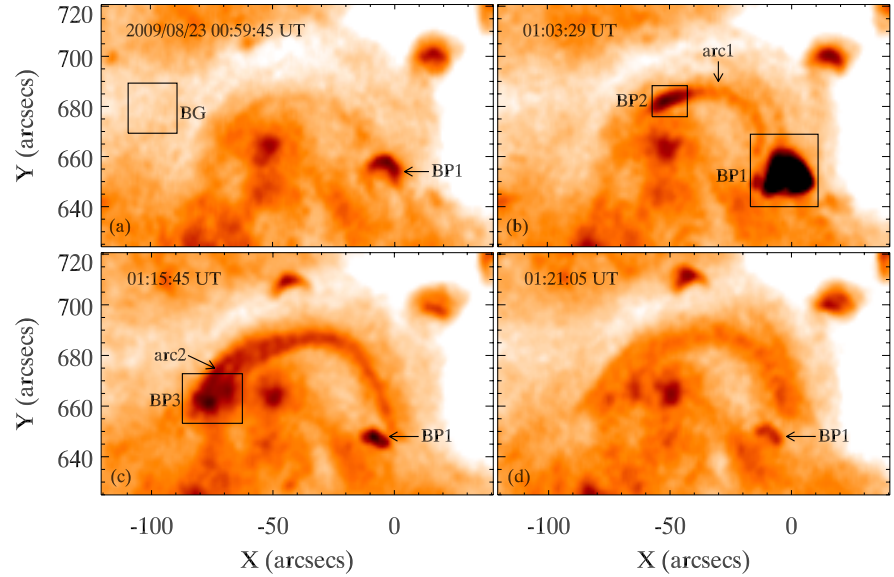

Fig. 1. Four snapshots of the SXR images observed by the XRT C_poly filter. BG in panel a) denotes the quiescent background region. BP1 represents the primary $\mathrm{CBP}$. $\mathrm{BP} 2$ in panel b) and $\mathrm{BP} 3$ in panel $c$ ) stand for the sympathetic CBPs induced by BP1. Arc1 in panel b) is the loop connecting BP1 and BP2. Arc2 in panel c) begins to develop in BP3. Note that the color is reversed.

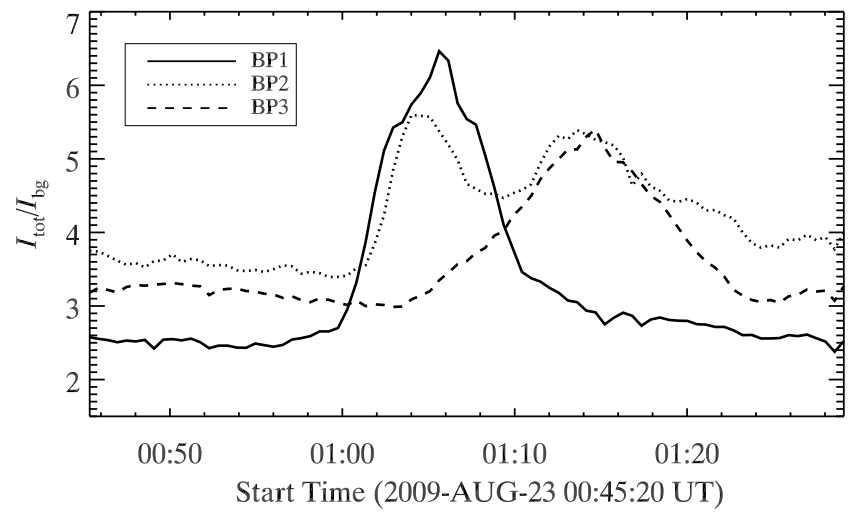

Fig. 2. SXR light curves of the three CBPs: BP1 (solid line), BP2 (dotted line), and BP3 (dashed line). $I_{\mathrm{tot}}$ and $I_{\mathrm{bg}}$ denote the total intensities of the CBPs and the background region. The intensities of BP2 and BP3 are multiplied by 6 and 2 to get a better comparison with BP1.

01:06 UT, showing a strong flash in SXR that resembles a microflare (Fig. 1b). Meanwhile, the brightening propagated from $\mathrm{BP} 1$ to a remote northeast region that is $\sim 60^{\prime \prime}$ away, producing another bright point (hereafter BP2) that is $\sim 15^{\prime \prime}$ long and much fainter than BP1. The faint loop connecting BP1 and BP2 is labeled with arc1, whose length is estimated to be 94" assuming a semi-circular shape. Parallel to arc1, there is another arc that also starts in BP1. Jet-like motion along the arc is observed. Before BP1 faded away, the third bright point (hereafter BP3) appeared and brightened $80^{\prime \prime}$ east of BP1 (Fig. 1c). It is 20" in size and is more diffused than BP1. Interestingly, BP3 is not isolated, but is probably connected to BP1 by arc 2 in the panel. We speculate that the arc parallel to arc1 is the right segment of arc2. After the transient brightenings that lasted for $\sim 30 \mathrm{~min}$, the three bright points and two arcs gradually disappeared in the SXR images (Fig. 1d).

Figure 2 displays the light curves of BP1 (solid line), BP2 (dotted line), and BP3 (dashed line) where $I_{\text {tot }}$ and $I_{\mathrm{bg}}$ denote the total intensities of the CBPs and the background region. Therefore, $I_{\mathrm{tot}} / I_{\mathrm{bg}}$ signifies background-normalized intensity. Since BP2 and BP3 are weaker than BP1, we multiply the 


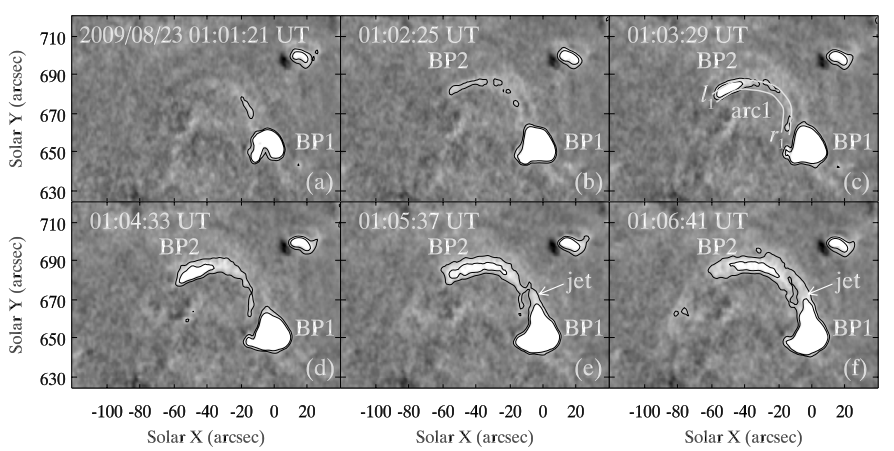

Fig. 3. Six snapshots of the base-difference images. The white/black color indicates enhancement/decrease in brightness. The black contours correspond to intensities of 25 and $50 \mathrm{DN} \mathrm{s}^{-1}$. In panel c), arc1 outlined by a pair of white parallel lines connects the two endpoints, $l_{1}$ in BP2 and $r_{1}$ in BP1. In panels e) and f), the arrows point to the upward jet-like motion originating from BP1.

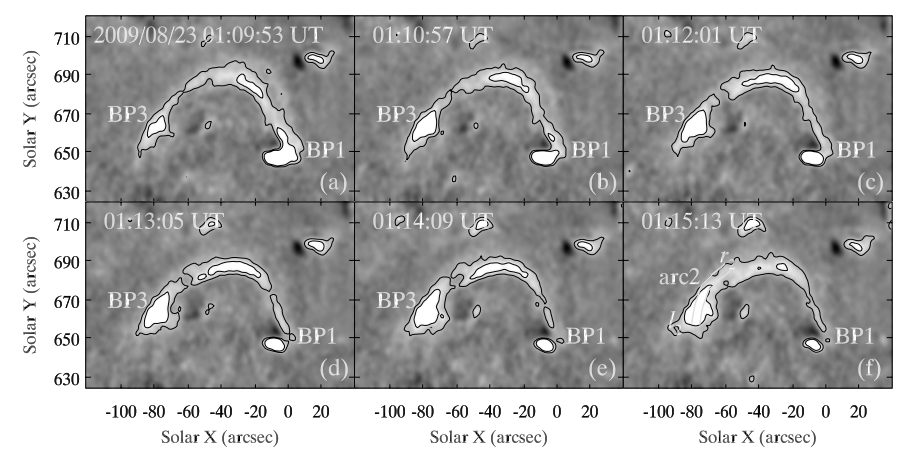

Fig. 4. Same as Fig. 3. In panel f), arc2 outlined by a pair of white parallel lines connects the two endpoints $l_{2}$ and $r_{2}$.

values of $I_{\text {tot }} / I_{\text {bg }}$ by 6 and 2 for BP2 and BP3 to get a clearer comparison. It is revealed that the intensity of BP1 increases gradually from $\sim 00: 56$ UT to $\sim 01: 00$ UT before a sharp rise until the maximum at $\sim 01: 06$ UT. Then, it declines rapidly to the value before the onset of flash. The intensity of BP3 starts to grow at $\sim 01: 03$ UT and reaches the apex at $\sim 01: 15$ UT before recovering to a low level at $\sim 01: 25$ UT. The maximum of BP3 is delayed by $\sim 9$ min compared to BP1. The intensity of BP2 begins to rise gently at $\sim 01: 00$ UT and peaks at $\sim 01: 04$ UT before declining to a lower level. The apparent second peak at $\sim 01: 14$ UT is due to the brightening of arc2 that lies in front of BP2. Afterwards, it decays slowly until 01:25 UT.

Albeit small compared to the flaring arcades (McKenzie \& Hudson 1999), we discovered the signature of chromospheric evaporation along arc1 and $\operatorname{arc} 2$. To show the evaporation more obviously, we take the first image at 00:45:20 UT on August 23 as the base image before performing base-difference for the others. Figure 3 displays six snapshots of the base-difference images where the colors white/black mean intensity enhancement/decrease. The black contours correspond to intensities of 25 and $50 \mathrm{DN} \mathrm{s}^{-1}$. In Fig. 3c, a pair of white parallel curves connecting $l_{1}$ and $r_{1}$ outline arc 1 . It is seen from the upper panels that the brightening propagates from $r_{1}$ to $l_{1}$ along the arc. The brightening does not remain at a constant place at $l_{1}$, i.e., BP2. On the contrary, it propagates backwards after the brightness of $\mathrm{BP} 2$ reaches maximum at $\sim 01: 04 \mathrm{UT}$, which is illustrated in the lower panels of Fig. 3.

As in Fig. 3, we demonstrate six snapshots of the basedifference images in Fig. 4. Likewise, we outline arc2 with a
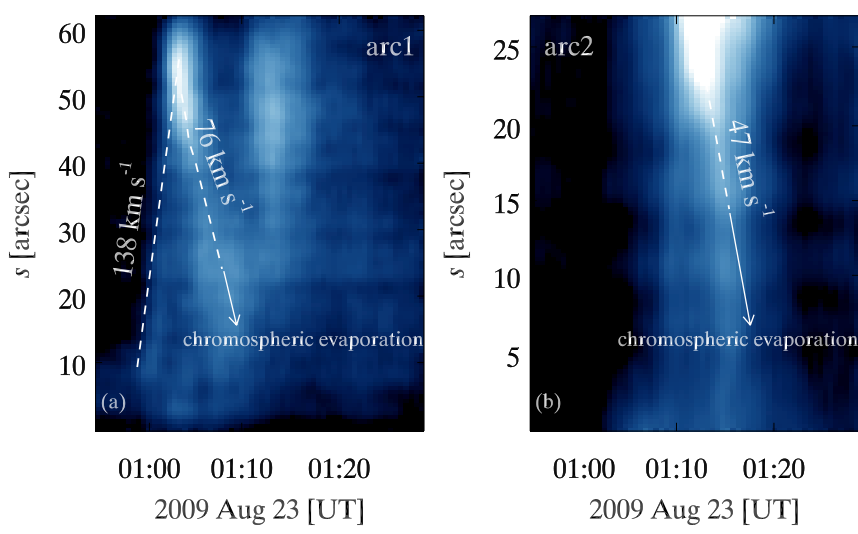

Fig. 5. Temporal evolutions of the SXR intensities of arc1 (left) and arc2 (right) labeled in Fig. 3c and Fig. 4f, respectively. The start points of coordinate $s$ in the $y$-axes are the right endpoints of $\operatorname{arc} 1$ and $\operatorname{arc2}$, i.e., $r_{1}$ and $r_{2}$. The slopes of the white dashed lines stand for the apparent velocities of the propagation of brightening along the arcs. The arrows point to the features of chromospheric evaporation.

pair of white parallel curves connecting $l_{2}$ and $r_{2}$ in Fig. $4 \mathrm{f}$. It is seen that as BP3 increases in size and brightness, the brightening propagates from $l_{2}$ to $r_{2}$ along arc 2 during 01:10 UT-01:15 UT, a clear signature of chromospheric evaporation.

To calculate the apparent velocities of the propagations of the brightenings along the arcs, we derive the temporal evolutions of SXR intensity of the arcs marked in Fig. $3 \mathrm{c}$ and Fig. $4 \mathrm{f}$ using the standard program plot_arc.pro in the Solar Software. The timeslice diagrams are presented in Fig. 5, where $s$ denotes the distances from the right endpoints of the arcs. In the left panel, it seems that the brightening propagates from near the right endpoint during 00:58 UT-01:03 UT, which is outlined by the white dashed line. The intensity contrast between this feature and the background levels elsewhere is quite low. The slope of the dashed line provides a rough estimation of the apparent velocity of the propagation, i.e., $138 \mathrm{~km} \mathrm{~s}^{-1}$. Afterwards, the brightening propagates in the opposite direction during 01:03 UT-01:07 UT. As we have mentioned before, it is an indication of chromospheric evaporation demonstrated in the lower panels of Fig. 3. The apparent velocity is estimated to be $76 \mathrm{~km} \mathrm{~s}^{-1}$. In the right panel, the evaporation occurs during 01:10 UT-01:15 UT at a speed of $\sim 47 \mathrm{~km} \mathrm{~s}^{-1}$.

\section{Discussion and conclusion}

Several agents have been proposed in previous works for the mechanism of energy transport from the main flare/CBP to sympathetic flare/CBP. The most popular is nonthermal electrons accelerated in the main flares and guided by the large-scale coronal loops connecting the primary and remote regions (Tang \& Moore 1982; Nakajima et al. 1985; Martin \& Svestka 1988). The electrons produce chromospheric evaporation after penetrating into the dense chromosphere of the remote region. Another candidate of energy transport is thermal conduction at a speed of hundreds of $\mathrm{km} \mathrm{s}^{-1}$ due to the super-hot plasma created by the primary flares (Rust et al. 1985; Bastian \& Gary 1992). The third agent is hot jets guided by the coronal loops as a result of interaction between small emerging loops and large preexisting loops (Hanaoka 1996, 1997; Nishio et al. 1997).

In our case study of sympathetic CBPs, we found tentative evidence of propagation of brightening from BP1 to BP2 at a speed of $\sim 138 \mathrm{~km} \mathrm{~s}^{-1}$ that is far less than the typical velocity of 
energetic electrons but in the same order of magnitude as thermal conduction speed (Campbell 1984; Mandrini et al. 1996). Therefore, we postulate that BP2 is heated by thermal conduction. For BP3, it is seen from Fig. 2 that its onset time lags behind that of BP1 by $\sim 4 \mathrm{~min}$, and the peak time of its intensity is delayed by $\sim 9$ min. However, definite propagation of brightening from BP1 to BP3 is not well observed except the jet-like motion guided by the arc parallel to arc1 prior to the onset of evaporation from BP3, as shown in Figs. 3e and 3f. If BP3 is heated by thermal conduction, the velocity is estimated to be $\sim 380 \mathrm{~km} \mathrm{~s}^{-1}$.

Although chromospheric evaporation has been extensively observed and investigated, direct evidence of the dynamic process is rare in SXR. Nitta (2012) recently reported the detection of chromospheric evaporation upflows in transient brightening events. The apparent velocities are up to hundreds of $\mathrm{km} \mathrm{s}^{-1}$. In our case, the velocities are $76 \mathrm{~km} \mathrm{~s}^{-1}$ and $47 \mathrm{~km} \mathrm{~s}^{-1}$ for BP2 and $\mathrm{BP} 3$, respectively. Considering the velocities of evaporation in the sympathetic CBPs, we conclude that they belong to the gentle type. Milligan (2008) studied a B-class flare. There was no detectable HXR emission in the flare ribbons, and the blueshift was very weak $\left(\sim 14 \mathrm{~km} \mathrm{~s}^{-1}\right)$. Considering the apparent flow of hot material along the flare loop, the author tentatively proposed that it was a signature of gentle evaporation caused by thermal conduction. We observed apparent evaporation flows of hot plasma along arc1 and arc2 that connect BP1 with BP2 and $\mathrm{BP} 3$, respectively. We also present tentative evidence of a thermal conduction front.

In this letter, we report our first discovery of sympathetic CBPs and the chromospheric evaporation observed by Hinode/XRT on 2009 August 23. A strong flash occurred in the primary $\mathrm{CBP}$, i.e., $\mathrm{BP} 1$, triggering the appearance of two adjacent CBPs, i.e., BP2 and BP3 that are $60^{\prime \prime}$ and $80^{\prime \prime}$ away from $\mathrm{BP} 1$, respectively. The peak time of $\mathrm{BP} 2$ intensity coincides roughly with that of BP1, but the peak time of BP3 intensity was delayed by $\sim 9$ min compared to BP1. The SXR brightening propagates from BP1 to BP2 along arc1 at a speed of $\sim 138 \mathrm{~km} \mathrm{~s}^{-1}$ followed by chromospheric evaporation in BP2 at a speed of $\sim 76 \mathrm{~km} \mathrm{~s}^{-1}$. The propagation of brightening from BP1 to BP3 was not well identified except the jet-like motion along the arc parallel to arc1. Nevertheless, we found chromospheric evaporation at a speed of $\sim 47 \mathrm{~km} \mathrm{~s}^{-1}$ in BP3. We propose that the gentle evaporation in $\mathrm{BP} 2$ and $\mathrm{BP} 3$ were generated by thermal conduction. Additional case studies using highcadence and high-resolution multi-wavelength observations are expected in the future. Numerical modeling would be helpful in trying to reproduce the observed upflows in response to a thermal conduction front.

Acknowledgements. The authors are grateful to the referee for enlightening comments and suggestions that improved the quality of our work. Q. M. Zhang acknowledges P. F. Chen, C. Fang, M. D. Ding, H. Peter, J. X. Wang, W. Q. Gan, Y. P. Li, S. M. Liu, L. Feng, H. Li, and Z. J. Ning for valuable discussions and suggestions. Q. M. Zhang also thanks Centre for mathematical Plasma Astrophysics at the Mathematics Department of the KU Leuven for the hospitality during his visit in June 2013. Hinode is a Japanese mission developed and launched by ISAS/JAXA, with NAOJ as domestic partner, and NASA and STFC (UK) as international partners. The research is supported by the Chinese foundations NSFC (11025314, 10878002, 10933003, and 11173062) and 2011CB811402.

\section{References}

Allred, J. C., Hawley, S. L., Abbett, W. P., \& Carlsson, M. 2005, ApJ, 630, 573 Bastian, T. S., \& Gary, D. E. 1992, Sol. Phys., 139, 357

Berkebile-Stoiser, S., Gömöry, P., Veronig, A. M., Rybák, J., \& Sütterlin, P. 2009, A\&A, 505, 811

Brosius, J. W., \& Holman, G. D. 2007, ApJ, 659, L73

Brosius, J. W., \& Holman, G. D. 2009, ApJ, 692, 492

Brosius, J. W., \& Phillips, K. J. H. 2004, ApJ, 613, 580

Campbell, P. M. 1984, Phys. Rev. A, 30, 365

Carmichael, H. 1964, NASA Sp. Publ., 50, 45

Chen, P. F. 2012, Hinode-3: The 3rd Hinode Science Meeting, eds. T. Sekii, T. Watanabe, \& T. Sahurai, ASP Conf. Ser., 454, 265

Chen, F., \& Ding, M. D. 2010, ApJ, 724, 640

Deng, N., Tritschler, A., Jing, J., et al. 2013, ApJ, 769, 112

Ding, M. D., \& Fang, C. 2001, MNRAS, 326, 943

Emslie, A. G., Li, P., \& Mariska, J. T. 1992, ApJ, 399, 714

Fisher, G. H., Canfield, R. C., \& McClymont, A. N. 1985a, ApJ, 289, 414

Fisher, G. H., Canfield, R. C., \& McClymont, A. N. 1985b, ApJ, 289, 425

Fisher, G. H., Canfield, R. C., \& McClymont, A. N. 1985c, ApJ, 289, 434

Fletcher, L., Dennis, B. R., Hudson, H. S., et al. 2011, Space Sci. Rev., 159, 19

Gan, W. Q., Zhang, H. Q., \& Fang, C. 1991, A\&A, 241, 618

Golub, L., Krieger, A. S., Harvey, J. W., \& Vaiana, G. S. 1977, Sol. Phys., 53, 111

Golub, L., Deluce, E., Austin, G., et al. 2007, Sol. Phys., 243, 63

Guo, Y., Ding, M. D., Wiegelmann, T., \& Li, H. 2008, ApJ, 679, 1629

Hanaoka, Y. 1996, Sol. Phys., 165, 275

Hanaoka, Y. 1997, Sol. Phys., 173, 319

Hao, Q., Guo, Y., Dai, Y., et al. 2012, A\&A, 544, L17

Hirayama, T. 1974, Sol. Phys., 34, 323

Ji, H., Huang, G., Wang, H., et al. 2006, ApJ, 636, L173

Ji, H., Huang, G., \& Wang, H. 2007, ApJ, 660, 893

Ji, H., Wang, H., Liu, C., \& Dennis, B. R. 2008, ApJ, 680, 734

Kopp, R. A., \& Pneuman, G. W. 1976, Sol. Phys., 50, 85

Kosugi, T., Matsuzahi, M., Sakao, et al. 2007, Sol. Phys., 243, 3

Li, Y., \& Ding, M. D. 2011, ApJ, 727, 98

Liu, W., Liu, S., Jiang, Y. W., \& Petrosian, V. 2006, ApJ, 649, 1124

Machado, M. E., Xiao, Y. C., Wu, S. T., Prokakis, T., \& Dialetis, D. 1988, ApJ, 326,451

Mandrini, C. H., Démoulin, P., van Driel-Gesztelyi, L., et al. 1996, Sol. Phys., 168,115

Martin, S. F., \& Svestka, Z. F. 1988, Sol. Phys., 116, 91

McKenzie, D. E., \& Hudson, H. S. 1999, ApJ, 519, L93

Milligan, R. O. 2008, ApJ, 680, L157

Milligan, R. O., Gallagher, P. T., Mathioudakis, M., \& Keenan, F. P. 2006, ApJ, 642, L169

Nakajima, H., Dennis, B. R., Hoyng, P., et al. 1985, ApJ, 288, 806

Neupert, W. M. 1968, ApJ, 153, L59

Ning, Z., \& Cao, W. 2011, Sol. Phys., 269, 283

Ning, Z., Cao, W., Huang, J., et al. 2009, ApJ, 699, 15

Nishio, M., Yaji, K., Kosugi, T., Nakajima, H., \& Sakurai, T. 1997, ApJ, 489, 976

Nitta, S., Imada, S., \& Yamamoto, T. T. 2012, Sol. Phys., 276, 183

Priest, E. R., Parnell, C. E., \& Martin, S. F. 1994, ApJ, 427, 459

Richardson, R. S. 1951, ApJ, 114, 356

Rust, D. M., Simnett, G. M., \& Smith, D. F. 1985, ApJ, 288, 401

Shibata, K., \& Magara, T. 2011, Liv. Rev. Sol. Phys., 8, 6

Silva, A. V. R., Wang, H., Gary, D. E., Nitta, N., \& Zirin, H. 1997, ApJ, 481, 978 Sturrock, P. A. 1966, Nature, 211, 695

Su, Y., Veronig, A. M., Holman, G. D., et al. 2013, Nature Phys., in press [arXiv: 1307.4527]

Svestka, Z. 1976, Solar Flares (Berlin, Heidelberg: Springer-Verlag), 415

Tang, F., \& Moore, R. L. 1982, Sol. Phys., 77, 263

Tian, H., Xia, L.-D., \& Li, S. 2008, A\&A, 489, 741

Wang, H. 2005, ApJ, 618, 1012

Wang, H., \& Liu, C. 2012, ApJ, 760, 101

Zhang, C., Wang, H., Wang, J., \& Yan, Y. 2000, Sol. Phys., 195, 135

Zhang, Q. M., Chen, P. F., Guo, Y., Fang, C., \& Ding, M. D. 2012, ApJ, 746, 19 